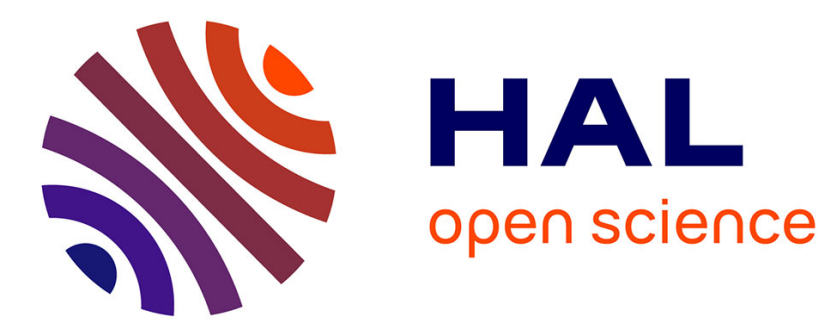

\title{
Comportement des matériaux à voiles de bateau
}

\author{
Vanessa Bohé, Pascal Casari, Françoise Léné, Peter Davies
}

\section{To cite this version:}

Vanessa Bohé, Pascal Casari, Françoise Léné, Peter Davies. Comportement des matériaux à voiles de bateau. Revue des composites et des matériaux avancés = Journal of Composite and Advanced Materials, 2003, 13 (3), pp.251 - 261. 10.3166/rcma.13.251-261 . hal-01005983

\section{HAL Id: hal-01005983 \\ https://hal.science/hal-01005983}

Submitted on 22 Oct 2016

HAL is a multi-disciplinary open access archive for the deposit and dissemination of scientific research documents, whether they are published or not. The documents may come from teaching and research institutions in France or abroad, or from public or private research centers.
L'archive ouverte pluridisciplinaire HAL, est destinée au dépôt et à la diffusion de documents scientifiques de niveau recherche, publiés ou non, émanant des établissements d'enseignement et de recherche français ou étrangers, des laboratoires publics ou privés. 


\title{
Comportement des matériaux à voiles de bateau
}

\section{Vanessa Bohé* - Pascal Casari** _ Françoise Léné*** \\ Peter Davies****}

\author{
* Voilerie Incidences la Rochelle \\ 8 rue de la désirée \\ F-17000 La Rochelle \\ vanessa@incidences-sails.com \\ ** GeM, Université de Nantes \\ 2 rue de la Houssinière BP9220,8 \\ F-44322 Nantes cedex 03 \\ Pascal.Casari@physique.univ-nantes.fr \\ ***LM2S, Université Paris 6 \\ Université Pierre et Marie Curie \\ 4 place Jussieu, F-75005 Paris \\ lene@ccr.jussieu.fr \\ ****IFREMER Centre de Brest \\ BP70, F-29280 Plouzané \\ Peter.davies@ifremer.fr
}

RÉSUMÉ. Les voiles de bateaux sont des surfaces portantes aérodynamiques. La stabilité de leur profil au cours des navigations et leur légèreté sont primordiales pour leur rendement. Les fibres de modules élevés sont donc largement utilisées pour la fabrication des matériaux à voiles. Ces propriétés mécaniques initiales ne sont pas toujours compatibles avec un bon vieillissement. Leur stabilité aux $U V$, au pliage, aux chocs et au fluage sont des facteurs limitants. Cette étude a pour objectif de qualifier les critères de performances des tissus laminés utilisés à la fabrication des voiles pour en extraire des voies de développement et d'optimisation.

ABSTRACT. Boat sails are aerodynamic surfaces. Their shape stability during sailing and their low weight are essential to their performance. High modulus fibres are widely used to make sails materials. Their initial mechanical properties are not always compatible with good ageing resistance. Their UV stability, flex, shock and creep resistance are limiting factors. The goal of this study is to qualify laminated sail fabric performance factors to extract ways to develop and optimise them.

MoTS-CLES : voile, matériaux, vieillissement.

KEYWORDS: sail, material, ageing. 


\section{Introduction}

Le gigantisme des nouveaux bateaux de course hauturière et la recherche constante de performance et de rendement des voiles sont à l'origine de l'émergence de nouvelles technologies comme le D4, le $3 D L$ ou le Cuben Fiber. Cet objectif de stabilisation des formes aérodynamiques au cours de la navigation implique l'utilisation de fibres de module de plus en plus élevé (aramides, UHMWPE, carbone, LCP). Ce gain de performance initial ne se fait pas sans contrepartie. Les gammes de vitesse de sollicitation des voiles (du fluage au choc) et les conditions d'utilisation (pliage, exposition aux UV, au faséiement, au milieu marin) s'ajoutent au cahier des charges. Cette étude a pour but de donner aux voileries des critères de choix des matériaux à voiles en fonction des conditions de navigation. Elle permet, conjointement, de définir des axes de développement et d'optimisation des performances.

\section{Les matériaux}

Les matériaux à voiles sont nombreux du fait de la diversité des fibres et des technologies utilisées. Nous avons donc limité cette étude aux tissus à voile dits "laminés » à fibres parallèles pour des commodités de test. Ils sont constitués de deux peaux extérieures en film polyester entre lesquelles sont laminées par collage des fibres distribuées suivant un réseau chaîne/trame $\left(0^{\circ}, 90^{\circ}\right)$. Cette technologie a l'avantage de pouvoir être appliquée à toutes les fibres utilisées en voilerie et d'être proche de celle des tissus émergents. Ces nouveaux matériaux, dits « orientés », sont issus de la volonté d'optimisation du rendement des voiles des unités de course (type Class America). Les fibres y sont distribuées parallèlement aux efforts. Leur dimensionnement et leur utilisation nécessitent, en amont, la compréhension du fonctionnement des tissus « laminés » classiques.

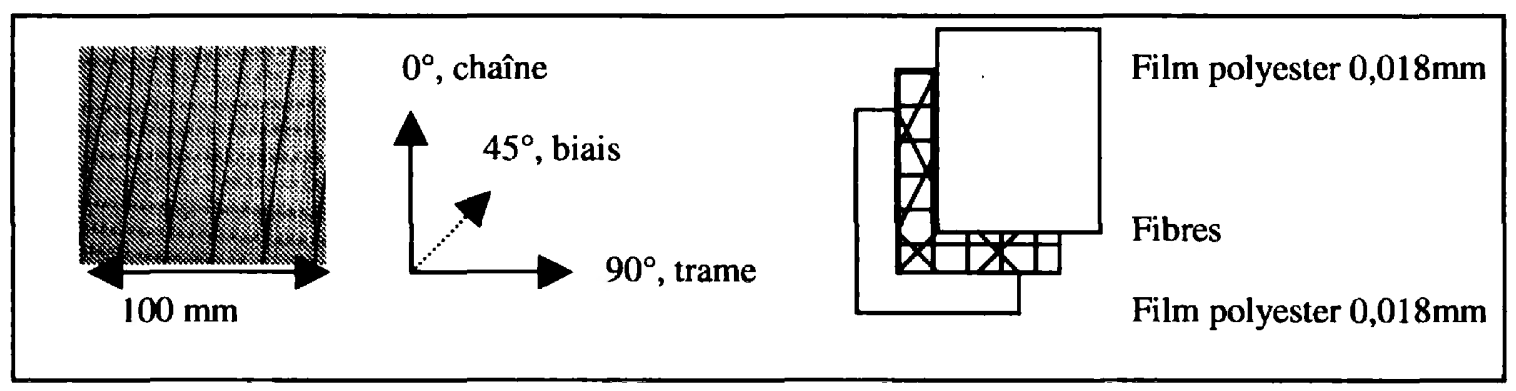

Figure 1. Tissu aramide laminé

Il ne s'agit pas dans ce travail de définir des valeurs de dimensionnement relatives au voilier considéré, mais d'établir des critères de performances intrinsèques aux matériaux essentiellement déterminés par les films, les fibres et l'interface de colle. 


\section{Les éléments constitutifs des tissus laminés}

Les trois principaux constituants des tissus à voile laminés sont le film, les fibres et la colle. Le film dans la plupart des cas est du polyester car il est peu onéreux, relativement stable au milieu extérieur, et de bonnes propriétés mécaniques. C'est lui qui assure l'étanchéité à l'air, la protection des fibres et la tenue dans le biais $\left(45^{\circ}\right)$ du tissu. La colle : il y a en fait deux colles. Une première qui est utilisée pour imprégner les fibres et la seconde pour le laminage. Elles ont pour rôle de garantir la cohésion du complexe, sa tenue au délaminage et de protéger les fibres du milieu extérieur. Cependant elles appartiennent au savoir-faire du fabricant de tissu. Il est fort difficile d'en connaître la nature. Les fibres utilisées sont essentiellement les carbones, aramides (twaron, kevlar), les polyester, les pentex, les UHMWPE (spectra, dyneema), les PBO (zylon) et les LCP (vectran). Leurs applications dépendent de leur module, de leur prix et de leur tenue au vieillissement.

\begin{tabular}{|c|c|c|c|c|c|c|c|c|}
\hline Fibre & Matériau & Fournisscur & $\begin{array}{l}\text { Module } \\
\text { GPa }\end{array}$ & $\begin{array}{l}\text { Ténacité } \\
\text { MPa }\end{array}$ & $\begin{array}{l}\text { Allongt. } \\
\text { à la } \\
\text { rupture }\end{array}$ & Utilisation & $\begin{array}{l}\text { Sensibilite } \\
\text { aux UV } \\
50 \% \\
\text { perte }\end{array}$ & $\begin{array}{l}\text { Flex, } \% \text { perte } \\
\text { après } 1 \text { cycle }\end{array}$ \\
\hline Dacron & Polyester & Dupont & 15 & 1000 & $15 \%$ & Croisière & 6 mois & Pas d'effet \\
\hline Pentex & Polyester & Honcywell & 30 & 1100 & $6 \%$ & Régate & 6 mois & Pas d'effet \\
\hline $\begin{array}{l}\text { Vectran } \\
\text { HS }\end{array}$ & $\begin{array}{l}\text { Polymère à } \\
\text { cristaux } \\
\text { liquides }\end{array}$ & $\begin{array}{l}\text { Hoechst } \\
\text { Cclanese }\end{array}$ & 73 & 3000 & $3.3 \%$ & Régate & $1-2$ mois & $15 \%$ \\
\hline $\begin{array}{c}\text { Twaron } \\
\text { HM }\end{array}$ & $\begin{array}{c}\text { Para- } \\
\text { Aramide }\end{array}$ & Teijin & 130 & 3000 & $2.9 \%$ & Régate & $2-3$ mois & $25 \%$ \\
\hline $\begin{array}{l}\text { Kevlar } \\
\text { Edge }\end{array}$ & $\begin{array}{l}\text { Para- } \\
\text { Aramide }\end{array}$ & Dupont & 130 & 3000 & $3 \%$ & Régate & $2-3$ mois & $25 \%$ \\
\hline Dynecma & UHMWPE & DSM & 120 & 2900 & $3.7 \%$ & $\begin{array}{c}\text { Course au } \\
\text { large }\end{array}$ & 6 mois & Pas d'effet \\
\hline $\begin{array}{c}\text { Spectra } \\
1000\end{array}$ & UHMWPE & Honeywell & 171 & 3300 & $2.7 \%$ & $\begin{array}{c}\text { Course au } \\
\text { large }\end{array}$ & 6 mois & Pas d'effet \\
\hline $\begin{array}{c}\text { Carbone } \\
\text { HR }\end{array}$ & $\begin{array}{l}\text { Carbone } \\
\text { PAN }\end{array}$ & Pyrofil & 230 & 4900 & $1.5 \%$ & Régate & $\begin{array}{l}\text { Pas } \\
\text { d'effet }\end{array}$ & $100 \%$ \\
\hline PBO & $\begin{array}{l}\text { Méta- } \\
\text { aramide }\end{array}$ & Toyobo & 250 & 3500 & $2.5 \%$ & Régate & 2 mois & $27 \%$ \\
\hline
\end{tabular}

Tableau 1. Principales fibres utilisées en voilerie

Le tableau 1 rappelle les propriétés génériques des fibres rencontrées en voilerie. Elles sont issues des données de Dimension Polyant (fournisseur de tissu), et vérifiées dans le cadre de ce travail. Les valeurs mécaniques ne tiennent pas compte de la viscoplasticité du matériau et le vieillissement est celui de la fibre nue. 


\section{Les essais mécaniques}

\subsection{Mesure de la gamme de vitesses de sollicitation in situ}

Des essais en mer ont été réalisés, afin de mieux connaître les sollicitations auxquelles sont soumises les voiles. Un extensomètre spécifique a été développé par P. Casari à l'Université de Nantes (Ducousso, 2002). Il est constitué d'un pont de Wheatstone collé sur une jauge en forme de $\mathrm{S}$ qui renvoie une tension corrélée à la déformation de la jauge par une courbe d'étalonnage. Cet outil de mesure est vissé en deux points sur la voile et relié par un câble à une centrale d'acquisition. Une première campagne d'essai réalisée sur une voile de croisière en tissé polyester nous a permis de mesurer une gamme de vitesses de sollicitation allant du fluage à $1 \mathrm{~s}^{-1}$, durant un empannage violent.

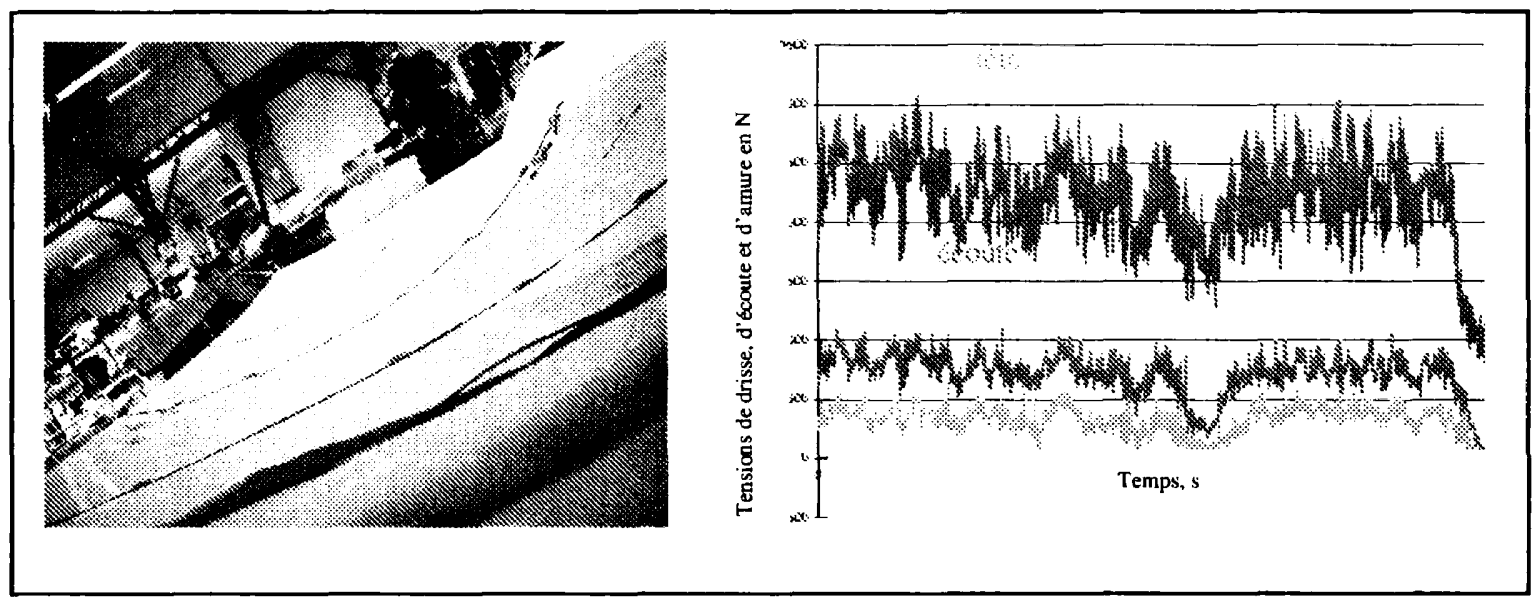

Figure 2. Disposition et étalonnage des jauges sur la voile et exemples de mesures

Lors d'une seconde campagne d'essai, un foc de «Melges 24» en laminé Pentex est instrumenté. L'objectif est de mesurer le chargement aux trois empointures et les valeurs de déformation le long d'un profil et de la chute, figure 2 . Les ordres de grandeurs mesurés aux trois coins (écoute, amure et tête) et sur la chute sont ceux attendus (Valette, 2001). Par contre, les mesures internes à la voile et sur le guindant ont posé des problèmes d'étalonnage. Ces zones sont assez creuses et difficiles à mettre en charge par simple mise sous tension des trois empointures.

\subsection{Etude du comportement mécanique des tissus}

Les essais in situ ont mis en évidence une gamme de vitesses de sollicitation assez vaste (du fluage à $1 \mathrm{~s}^{-1}$ ). Pour analyser le comportement des tissus à voile, trois types de tests mécaniques ont été considérés (I.T.F, 1995). 


\subsubsection{Essai de fluage}

Le fluage est réalisé sur une machine de traction MTS 25 asservie en force, équipée de mors de capacité 1 tonne à serrage mécanique. Des éprouvettes de tissu de $50 \mathrm{~mm} * 300 \mathrm{~mm}$ entre mors sont maintenues à une charge constante correspondant à $1 \%$ d'allongement (mesuré lors d'un essai à $50 \mathrm{~mm} / \mathrm{mn}$ ) pendant $12 \mathrm{~h}$, figure 3 . Si on élimine la partie de la courbe liée au freinage de la machine de traction après sa mise en charge, ces tests montrent deux zones principales. La première, très courte, est liée à la construction du tissu. Les fils de chaîne se tendent sur les fils de trame. La seconde est représentative du comportement au fluage de la fibre constituant le matériau testé.

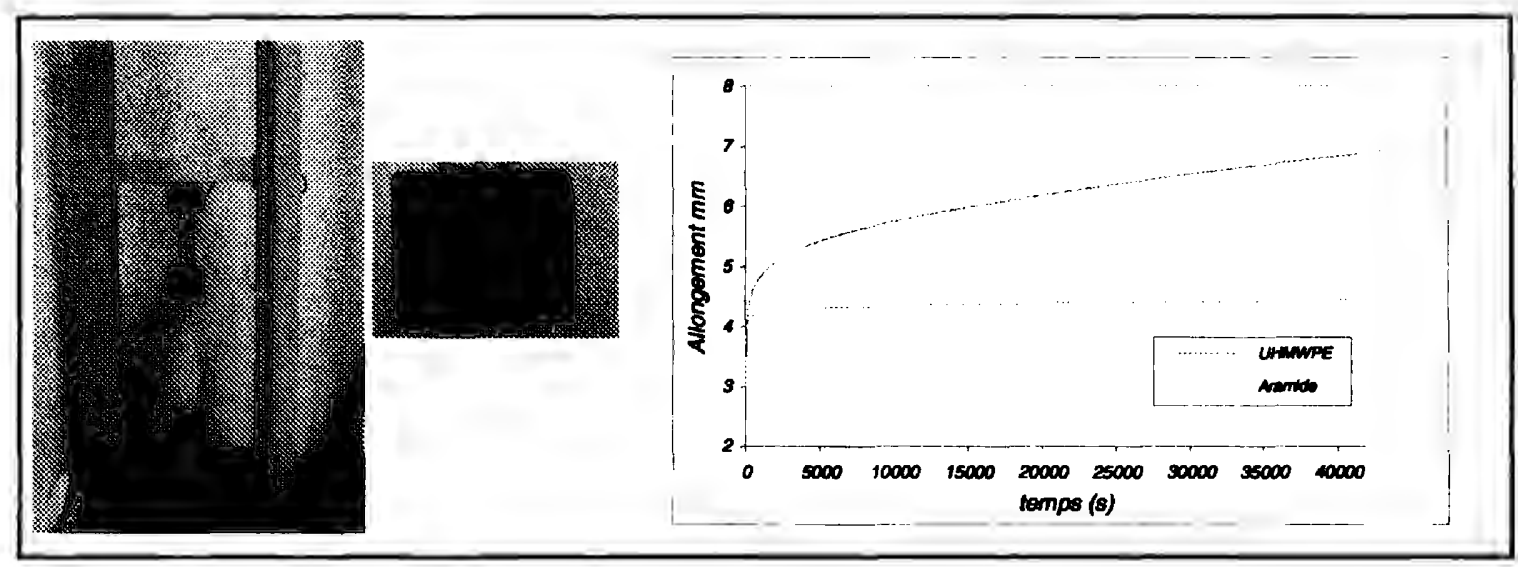

Figure 3. Dispositif d'essai en fluage et exemple de courbes obtenues

Le graphique de la figure 3 montre l'importance de la fibre dans la tenue de forme de la voile en navigation.

\subsubsection{Essais quasi statiques}

Les essais à vitesse moyenne, figure 4 , sont réalisés sur la même machine de traction mais asservie en déplacement. La géométrie des éprouvettes est conservée. L'objectif ultime sera d'écrire des lois simplifiées permettant de prédire les performances en module et en rupture des tissus à voile en fonction de la vitesse, du titre des fibres, de leur densité et du nombre de croisements, figure 5.

Les performances mécaniques des tissus (module et ténacité) sont mesurées dans les deux directions principales $\left(0^{\circ}, 90^{\circ}\right)$ et à des angles intermédiaires $\left(6^{\circ}, 30^{\circ} \mathrm{et}\right.$ $\left.45^{\circ}\right) .6^{\circ}$ représente l'angle d'ouverture moyen d'une laize de la voile, $45^{\circ}$ permet de caractériser la « matrice » constituée du film et de la colle, figure 6 , et $30^{\circ}$ est intermédiaire à ces deux valeurs. Les tests à $45^{\circ}$ montrent que le module en biais est quasiment celui d'un film polyester d'épaisseur égale à celle de la somme des deux peaux extérieures. Ce résultat corrobore les informations obtenues sur la colle de laminage : colle souple à plus de $500 \%$ d'allongement. Dans la suite de ce travail, l'interface entre les fibres est donc assimilée à un film polyester de $37,5 \mu \mathrm{m}$ 
d'épaisseur. La divergence des deux courbes autour de la plastification est dû au réarrangement des fibres dans le tissu à voile.

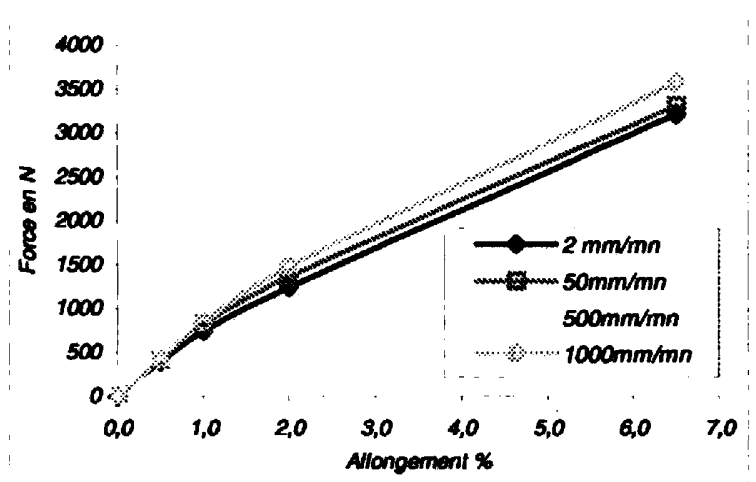

Figure 4. Evolution des propriétés du tissu Pentex en chaîne avec la vitesse de traction

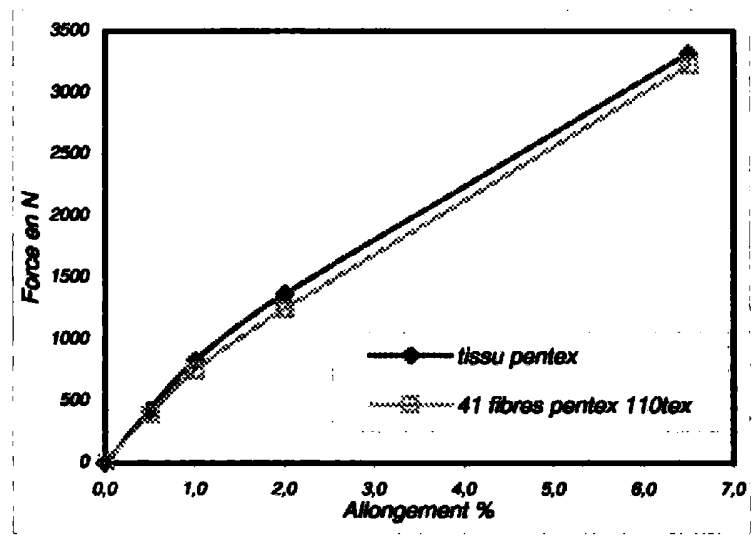

Figure 5. Comparaison des propriétés du tissu Pentex avec la réponse des fibres le constituant, à $50 \mathrm{~mm} / \mathrm{mn}$

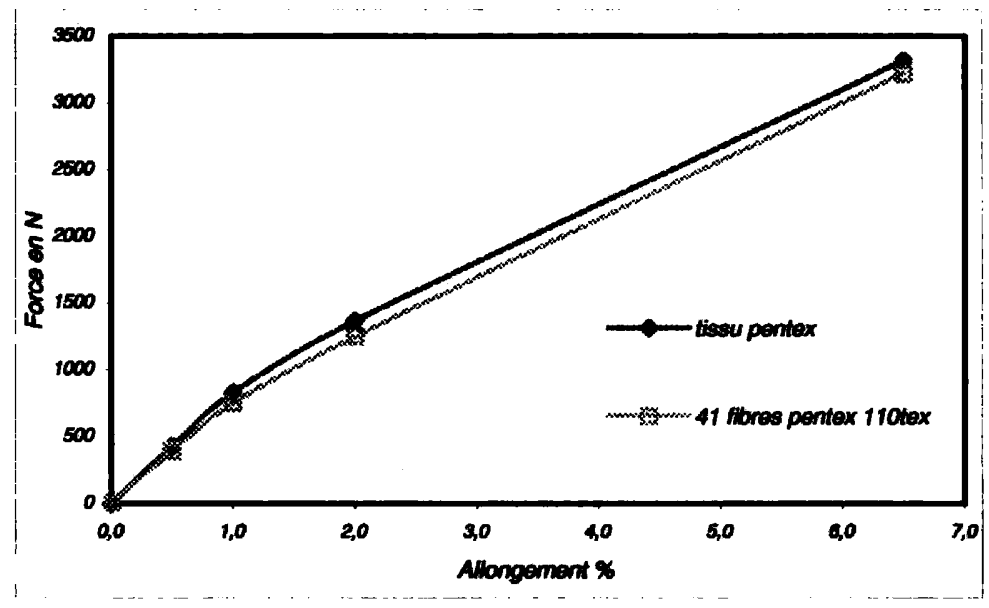

Figure 6. Comparaison de propriétés mécaniques du tissu pentex en biais et du film polyester $2 * 18,75 \mu \mathrm{m}$ constituant les peaux

\subsubsection{Les essais dynamiques}

Enfin pour atteindre des gammes de vitesse de $1 \mathrm{~s}^{-1}$, un montage spécifique est réalisé à l'Ifremer de Brest (Toullec, 2004). Des mors à rouleaux sont montés sur un vérin hydraulique pouvant se déplacer à $1000 \mathrm{~mm} / \mathrm{s}$, figure 7 . L'acquisition est réalisée à une vitesse de $100 \mathrm{kHz}$ et une caméra numérique à 4000 images/s filme les essais. Ce dispositif a permis de comparer l'évolution des valeurs de rupture des tissus pris dans le sens des fibres, avec la vitesse de traction. 


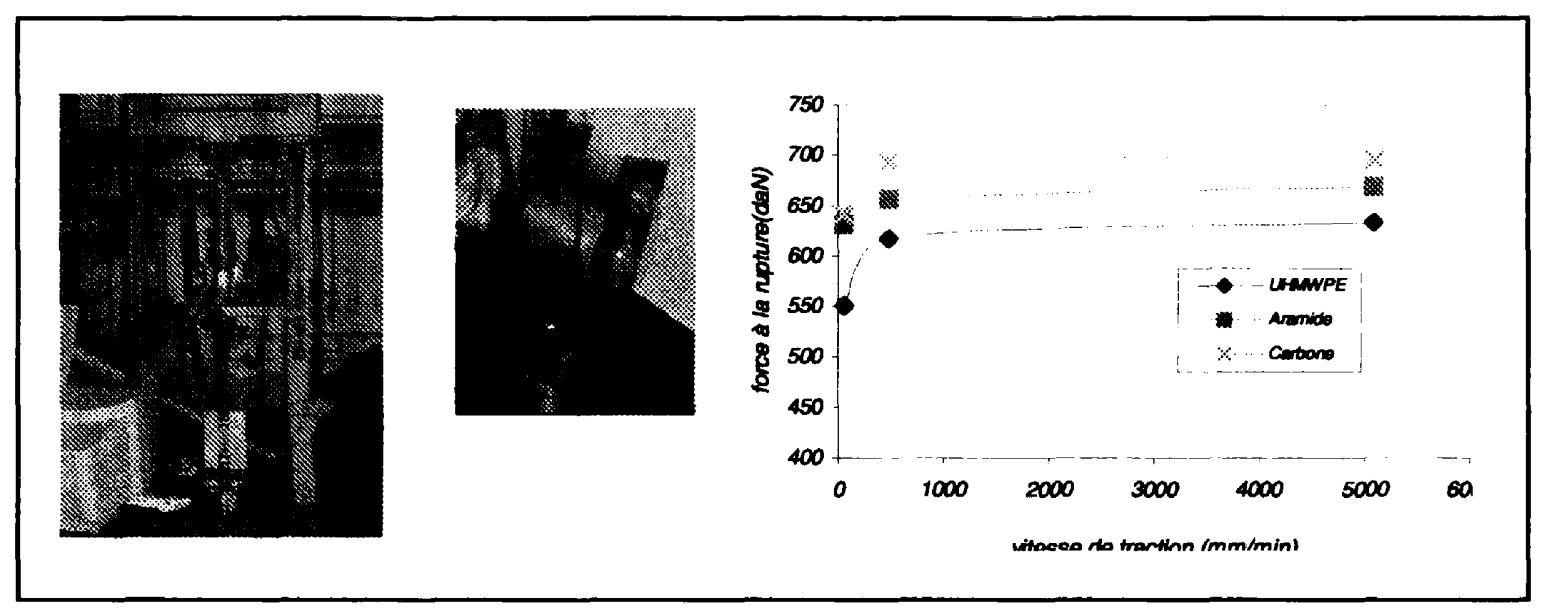

Figure 7. Dispositif de test à grande vitesse et résultats obtenus pour trois tissus

Des études de DMA (Analyse Dynamique Mécanique) sont également menées, afin de caractériser le comportement viscoélastique du film polyester. La figure 8 présente les résultats obtenus sur un film polyester de $175 \mu \mathrm{m}$. Ils montrent une forte dépendance de la vitesse de chargement autour de $\mathrm{Tg}=80^{\circ} \mathrm{C}$. Autour de $35^{\circ} \mathrm{C}$, température moyenne des voiles en été, la viscoélasticité est moins prononcée.

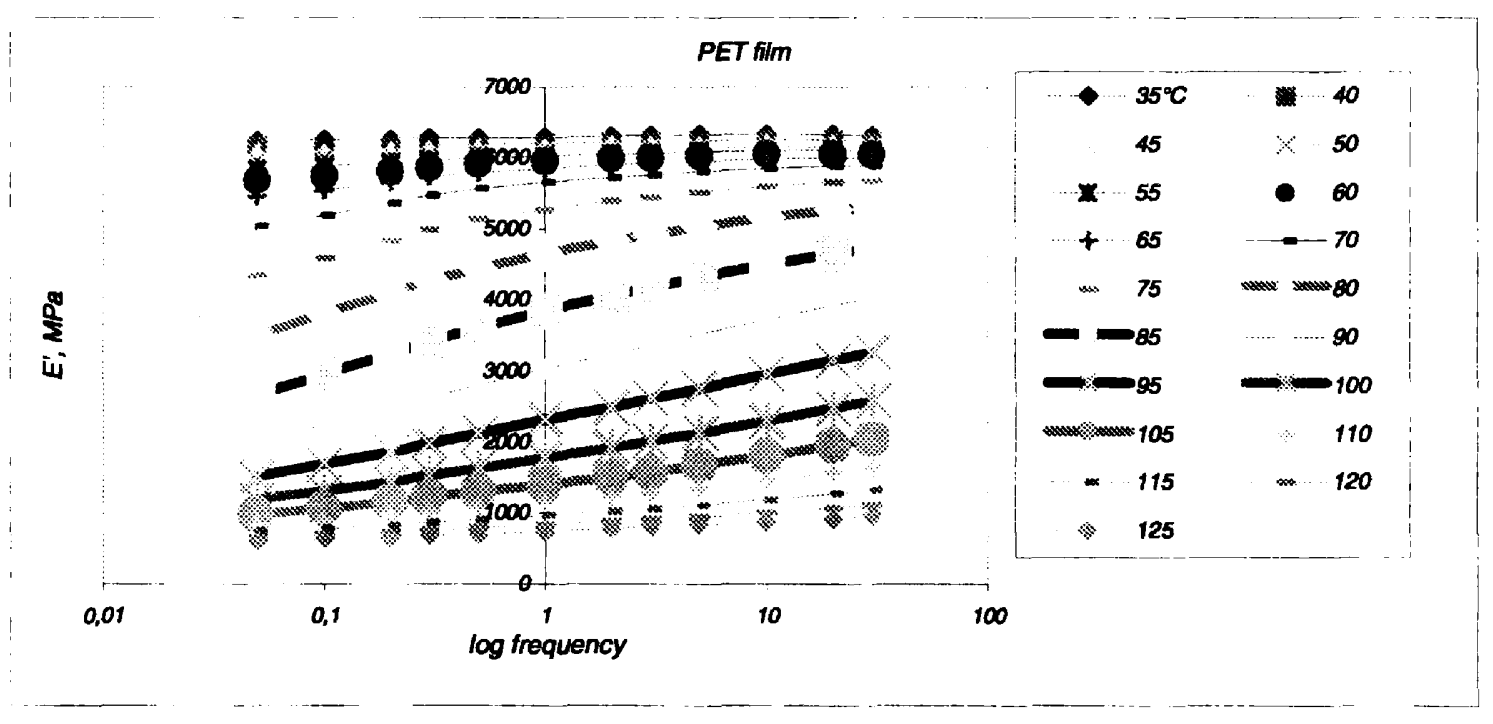

Figure 8. Evolution de la viscoélasticité de film polyester avec la température

\subsection{Les facteurs d'échelle}

L'objet de ce travail est d'appréhender le fonctionnement des matériaux au sein des voiles. Dans un premier temps la géométrie des éprouvettes est multipliée d'un facteur 10 dans les deux directions. Des mors spécifiques sont adaptés à une machine de traction horizontale de $100000 \mathrm{daN}$, asservie en déplacement. Le champ 
des déformations est suivi à l'aide d'une caméra numérique et un extensomètre mesure en temps réel le chargement (figure 9). Aujourd'hui, ce montage nous a permis de corroborer les mesures de module réalisées sur des éprouvettes de $50 \mathrm{~mm} * 300 \mathrm{~mm}$ et $500 \mathrm{~mm} * 3000 \mathrm{~mm}$ sur du film polyester de $175 \mu \mathrm{m}$. Des essais complémentaires seront menés sur un laminé aramide pris dans le sens des fibres. La comparaison des images numériques nous permettra de suivre les déformations de l'éprouvette au cours du chargement et de contrôler les zones d'influence des mors. Une fois ces tests validés, l'objectif est de passer sur des géométries plus complexes, représentatives de la forme de la voile.

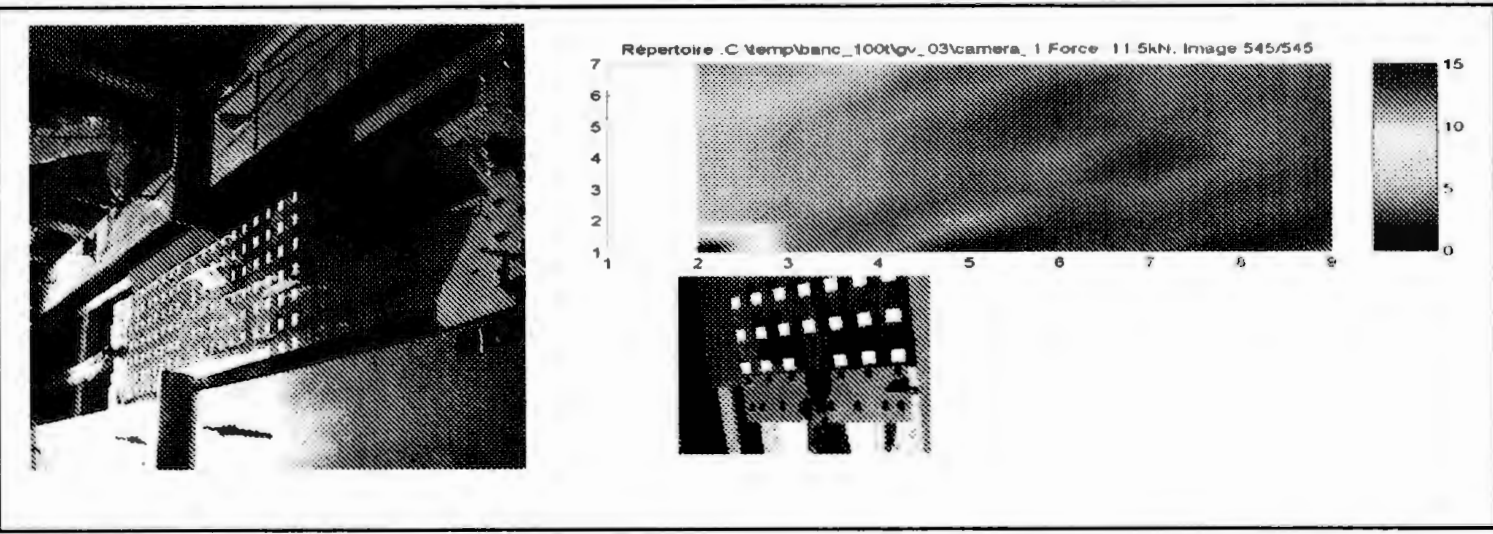

Figure 9. Dispositif de test en grande longueur et exemple de champs de déformation avant rupture

\section{Le vieillissement des voiles}

Le vieillissement des voiles n'est pas uniquement dû aux sollicitations mécaniques. Elles évoluent dans un milieu marin agressif (UV, salinité, humidité...) et sont fréquemment pliées ou froissées. L'expérience montre que le pliage, le choc et l'exposition aux UV et à l'humidité sont les facteurs principaux de dégradation des voiles.

\subsection{Le faséiement}

Le faséiement génère des chocs qui entraînent des micros fissures des films extérieurs laissant s'infiltrer l'eau et les UV. Lors des essais en mer, des vitesses de sollicitation de $2 \mathrm{~s}^{-1}$ ont été mesurées. Afin de rendre compte de la réalité, des échantillons de tissus ont été exposés au milieu extérieur durant 15 jours puis à une tempête durant 20 heures (figure 10). Les résultats obtenus sont comparables à ceux mesurés sur les chutes des voiles après une saison d'utilisation intense. 

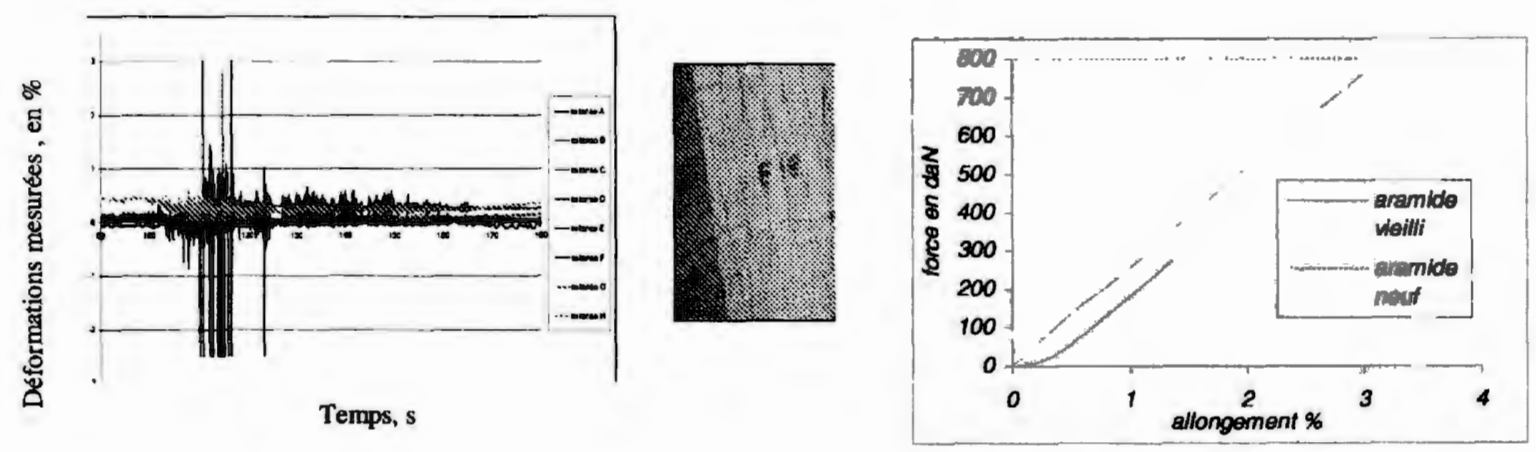

Figure 10. Déformations mesurées lors d'un faséiement, état et performances mécaniques du tissu après exposition à la tempête

\subsection{Le pliage}

Le gain de module en traction des fibres se fait généralement avec une perte d'allongement à la rupture. C'est le cas des fibres aramides ou de carbone. La résistance au pliage des composites en est parallèlement altérée. Le pliage des voiles est très contraignant. Les plis sont marqués par le bordé d'une bosse de ris, l'écrasement de la part d'un équipier ou par un enroulement. Les tissus sont donc testés dans cet esprit. Ils sont soumis à 10 plis marqués puis testés en traction à $50 \mathrm{~mm} / \mathrm{mn}$. Les valeurs de rupture sont alors comparées avec celles d'échantillons neufs. Il en ressort une stabilité au pliage liée à celle de la fibre constituant le matériau, au diamètre de celle-ci, à la nature et l'épaisseur des couches qui la recouvrent et à la technologie de fabrication du tissu.

\subsection{L'immersion}
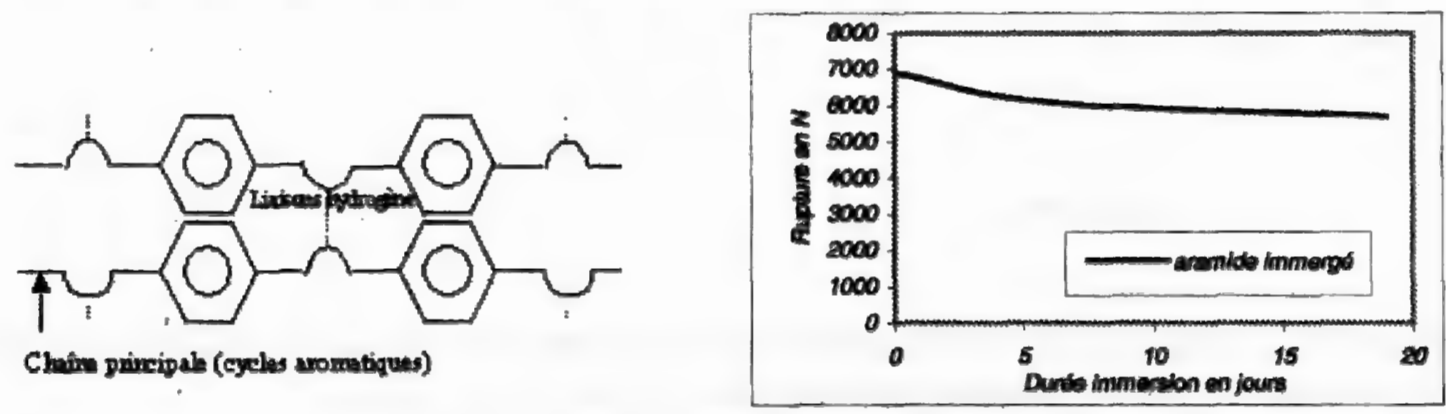

Figure 11. Vieillissement à l'immersion de l'aramide

Les tests d'immersion ont deux principaux objectifs : qualifier la perte de performances mécaniques des tissus et quantifier leur prise de poids. Certaines 
fibres, comme les aramides se dégradent au contact de l'eau. Les résultats obtenus sont liés aux matériaux constituant les tissus et à la technique de fabrication. Plus les fibres seront imprégnées, moins l'eau pourra se propager. Ce dernier point a fait l'objet de mesure par diffusion d'encre par gravité.

\subsection{Les $U V$}

Des essais de vieillissement accéléré aux rayonnements solaire ont été effectués dans l'enceinte climatique de L'Ifremer de Brest suivant la norme GAM EG 13, Fascicule 09. La lampe utilisée est à base d'halogénures métalliques, montée derrière un filtre borosilicate. Elle reproduit le spectre solaire à la surface de l'océan dans la gamme $280 \mathrm{~nm}-3000 \mathrm{~nm}$. Afin de valider ce choix des essais comparatifs ont été réalisés avec le concours de la société Atlas suivant plusieurs normes automobile pour les matériaux extérieurs : D27 1389, SAEJ 1960 (américaine). Cette étude a permis de comparer la stabilité des différentes fibres à l'ensoleillement. Les colles de laminage des tissus peuvent être chargées de $\mathrm{TiO} 2$ ou de benzotriazole et les films traités «anti-UV ». L'efficacité des protections est estimée pour chacune des fibres. Le passage du vieillissement en enceinte UV à celui en environnement naturel n'est pas trivial. L'exposition aux rayonnements solaires est cyclique et son intensité liée à de multiples facteurs comme la saison, la position géographique, la nébulosité, l'hygrométrie, la pollution, la présence de micro-organismes ou la température. Ainsi, des échantillons de même tissu sont exposés dans la cour de la voilerie pour des durées de 1 à 5 mois (figure 12). Enfin, tous ces tests sont mis en relation avec ceux réalisés sur des voiles carottées.

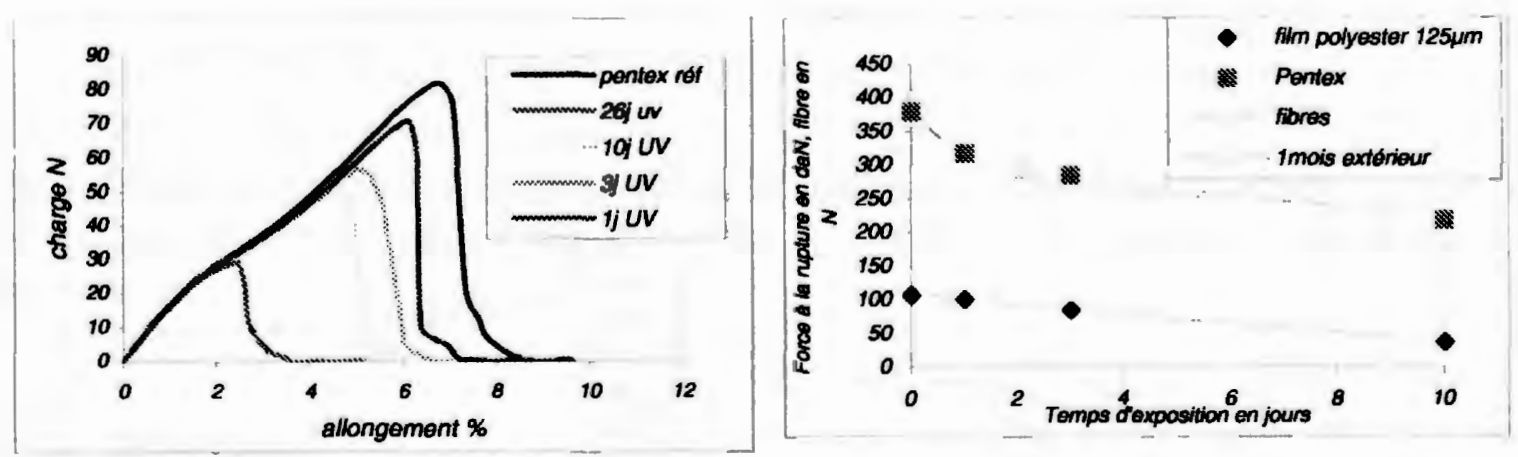

Figure 12. Influence du vieillissement sur le comportement en traction du pentex comparaison du vieillissement en enceinte et en milieu extérieur 


\section{Conclusion}

La performance des matériaux à voiles est liée à de multiples facteurs parfois antinomiques (module/vieillissement). Cet article a pour objectif de donner au lecteur une vision d'ensemble des paramètres agissant sur leur qualité. Des études complémentaires sont en cours pour définir des critères de performance en fonction des navigations envisagées, ainsi que des outils numériques de prédiction des déformations et d'optimisation de l'orientation des fibres dans des tissus laminés « orientés ». La prise en compte du vieillissement est difficile du fait du nombre de facteurs en interaction. Une analyse multicritère est envisagée.

\section{Bibliographie}

Ducousso N., Mesures de déformation des voiles, Rapport de stage ingénieur, Université de Nantes, 2002.

Institut Textile de France, Modalités de tests des tissus à voile, Etude réalisée pour le Groupe Incidences, ITF, 1995.

Toullec R., Etude des différents éléments constitutifs d'un bateau de compétition, stage ingénieur Ecole Centrale de Lyon, 2003.

Valette J., Simulation du comportement mécanique d'un ensemble voiles gréement de bateau, Thèse de doctorat Université d'Orléans, 2001. 